\title{
Ocular adverse events from pharmacological treatment in patients with multiple sclerosis-A systematic review of the literature
}

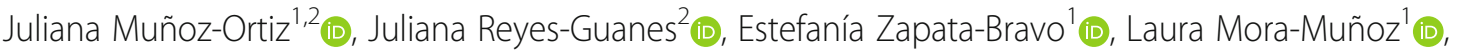 \\ Juan Antonio Reyes-Hurtado ${ }^{1}$ (D) Luis Octavio Tierradentro-García ${ }^{1}$ (D), William Rojas-Carabali ${ }^{1}$ (D), \\ Marcela Gómez-Suarez ${ }^{2}$ (I) and Alejandra de-la-Torre ${ }^{1 *}$ (D)
}

\begin{abstract}
Purpose: The aim of this study was to review the scientific evidence and describe the ocular treatment-emergent adverse events (TEAEs) related to pharmacological treatment in patients with multiple sclerosis.

Methods: A systematic review of literature was conducted according to the Preferred Reporting Items for Systematic Reviews and Meta-analysis guidelines in the MEDLINE, LILACS, EMBASE, and COCHRANE databases. Articles were filtered based on title and abstract considering the selection criteria and subsequently filtered by fulltext reading. The resulting articles were evaluated using the Joanna Briggs Institute Quality Tools. Study characteristics and results were extracted and presented in structured tables to conduct a narrative synthesis.

Results: A total of 2852 published articles were extracted using our strategy. After removing duplicates, 2841 articles were screened based on title and abstract, 102 articles were evaluated using quality tools, and 69 articles were filtered by full-text reading. Through this search strategy, 60 articles met all the inclusion criteria and seven articles, through a search update conducted in the same manner, were included. This resulted in 67 articles meeting the inclusion criteria, of which 11 were experimental and 56 were observational. The therapies related to ocular TEAEs were alemtuzumab, amantadine, fingolimod, steroids, CTLA-4 Ig, estriol, interferon $\beta$, natalizumab, hyperbaric oxygen, rituximab, siponimod, teriflunomide, and tovaxin. Fingolimod and siponimod were commonly associated with macular edema, interferon $\beta$ was associated with retinopathy, alemtuzumab was associated with thyroid eye disease, amantadine was associated with corneal edema, and steroids were associated with acute retinal necrosis. Opportunistic infections were also found, and there was one life-threatening case.
\end{abstract}

Conclusions: Our search revealed different methodological assessments of the topic. However, longitudinal studies regarding ocular TEAEs related to multiple sclerosis therapy are necessary to provide evidence-based recommendations, especially in understudied regions such as Latin America and Africa. Physicians should monitor ocular symptoms in patients being treated for multiple sclerosis and consider an interdisciplinary approach.

\footnotetext{
* Correspondence: alejadelatorre@yahoo.com

"Neuroscience Research Group "NeURos", Escuela de Medicina y Ciencias de la Salud, Universidad del Rosario, Carrera 24 \# 63C - 69, Bogotá, Colombia Full list of author information is available at the end of the article
}

C C The Author(s). 2021 Open Access This article is licensed under a Creative Commons Attribution 4.0 International License, which permits use, sharing, adaptation, distribution and reproduction in any medium or format, as long as you give appropriate credit to the original author(s) and the source, provide a link to the Creative Commons licence, and indicate if changes were made. The images or other third party material in this article are included in the article's Creative Commons licence, unless indicated otherwise in a credit line to the material. If material is not included in the article's Creative Commons licence and your intended use is not permitted by statutory regulation or exceeds the permitted use, you will need to obtain permission directly from the copyright holder. To view a copy of this licence, visit http://creativecommons.org/licenses/by/4.0/. The Creative Commons Public Domain Dedication waiver (http://creativecommons.org/publicdomain/zero/1.0/) applies to the data made available in this article, unless otherwise stated in a credit line to the data. 


\section{Systematic review registration: PROSPERO ID CRD42020106886}

Keywords: Drug-Related Side Effects and Adverse Reactions, Multiple Sclerosis, Therapeutics, Eye Diseases

\section{Key points}

- This study was conducted to review the scientific evidence and describe the ocular TEAEs related to pharmacological treatment in patients with multiple sclerosis to provide a theoretical basis and quality literature for ophthalmology specialists, neurologists, and general practitioners, which could help them in making appropriate clinical decisions.

- Patients under fingolimod and interferon $\beta$ treatment showed a higher frequency of TEAEs. Alemtuzumab was associated with an autoimmune response that manifested as thyroid eye disease.

- We recommend physicians to be cautious when treating patients with multiple sclerosis and monitor the ocular symptoms that patients may present. An interdisciplinary approach might be considered to evaluate the patient's requirements.

\section{Background}

Multiple sclerosis (MS) is a chronic autoimmune demyelinating disease with variable clinical, radiological, and pathological characteristics. Since its first description by Charcot, there have been significant efforts to clarify its pathophysiology and progression and determine the best strategies for appropriate management [1]. Currently, the therapy for MS comprises corticosteroids, immunosuppressants, and immunomodulators such as interferon (INF) and monoclonal antibodies. Although several cases of patients with ocular TEAEs have been reported over the years, there is a lack of clear understanding of the TEAEs that are strictly related to the disease and linked only to therapy [2].

Ocular manifestations in patients with MS are not rare and have been described as a major aspect of the disease. Any structure related to visual pathways could be involved in MS, and up to $20 \%$ of patients would display optic neuritis as an initial clinical manifestation. Visual field and color vision defects, relative afferent pupillary defect, and other ophthalmological findings could also be present [3].

According to the World Health Organization (WHO), the definition of drug-related adverse events corresponds to an involuntary harmful response to a medication used in a normal dose for a prophylactic, diagnostic, or therapeutic purpose $[4,5]$. However, it is necessary to consider that this harmful response, irrespective of how minor it is, must be explained to the patient before initiating any medication to predict, prevent, or treat any possible future event and avoid poor adherence to treatment [5]. Some of the TEAEs that have been described as related to therapy in MS are cataract, glaucoma, chorioretinopathy, visual acuity reduction, macular edema, retinopathy, among others [2].

The aim of this study was to review the scientific evidence and describe the ocular TEAEs related to pharmacological treatment in patients with MS to provide a theoretical basis and quality literature for ophthalmology specialists, neurologists, and general practitioners, which could help them in making appropriate clinical decisions.

Methods This review was written according to the Preferred Reporting Items for Systematic Reviews and Meta-analysis (PRISMA) extension statement for reporting systematic reviews. The protocol registration can be found under the PROSPERO ID CRD42020106886.

\section{Study design}

A systematic literature review focusing on ocular TEAEs in MS therapy was conducted using the MEDLINE, LILACS, EMBASE, and COCHRANE databases for articles published till November 2018. The search was updated on May 4, 2020. MEDLINE and COCHRANE databases were searched using Medical Subject Headings terms, EMBASE was searched using emtree terms, and LILACS was searched using Descriptores en Ciencias de la Salud terms, using boolean operators, as evidenced in Annex 1. We limited the search only to human studies, although no limits regarding language and period of publication were used.

\section{Study selection}

An initial search was conducted by JMO, JRG, LMM, and JARH, based on which a review was prepared to eliminate duplicates. Eligible studies were selected by screening the title and abstract by at least two reviewers independently, and discrepancies were resolved by an expert evaluator (ADLT). A study was included if (a) the abstract was available, (b) it contained original data, (c) the diagnosis of MS was made by a specialist, (d) the individual was being treated for MS, and (e) the ocular manifestations appeared after the initiation of MS treatment. Experimental and observational studies were also included. Articles were excluded from the analysis if they did not provide information regarding ocular TEAEs in individuals undergoing MS therapy. 
The full texts of the selected articles were retrieved and classified according to the type of article/study by at least two reviewers independently, and discrepancies were resolved by an expert evaluator (MGS). They were then evaluated using the Joanna Briggs Institute Quality Tools (JBIQT). If an article was not available, contact with the author was made. The cascade of article selection was managed using the PRISMA flowchart [6]. The following information was extracted from the selected articles: study period, location, number of patients, type of medication, and ocular TEAEs. A dataset was constructed using the information mentioned ahead.

\section{Data synthesis and presentation of results}

Microsoft Excel (Microsoft Corp., Redmond, WA, USA) was used as a synthesis tool to organize the results of the search strategy and the information of the articles. The first sheet contained information about the initial search results without duplicates. The next six sheets were asigned to three pairs of authors (LMM vs JARH, WRC vs JRG, and JMO vs LOTG) to filter titles and abstracts in a peered manner. A color code was used to select the articles as follows: green to include, yellow to revise, and red to exclude. The next three sheets were used to evaluate concordance for each pair of authors. The subsequent sheet was used by the expert reviewer (ADLT) to resolve discrepancies from the peered review. An additional sheet was used to classify the methodological design of the studies and record JBIQT grades and the reasons for exclusion. The next sheet was used to record articles included after full-text reading. The final sheet included information regarding ID number, authors, year of application of the study, year of publication, journal, location, title, aim, sample size, population (gender and group characteristics), methodological design, MS type, therapy, time of presentation of TEAEs, symptoms after therapy suspension, statistical analysis, important details from results, important details from discussion, and observations. The same synthesis tool was used for the search strategy update. The extracted data were synthetized in tables and figures to present them in an organized manner throughout the article.

\section{Risk of bias}

We retrieved and classified the articles based on study design to evaluate their methodological quality using their respective JBIQT [7]. Each quality tool has a set of questions that are evaluated as yes, no, unclear, or not applicable domains. An expert in epidemiology (MGS) established a minimum cut-off point as the number of items on the tool marked as "Yes," including the major and minor criteria. Case reports with 5/8 items on the checklist were included; for case series, the minimum cut-off point was 6/10 items; for cross-sectional studies, it was 6/8; for randomized controlled trials (RCTs), it was 10/13; and for nonrandomized experimental studies, it was 6/9 (see Annex 2 for supplementary information).

\section{Results}

\section{General description}

We extracted 2852 published articles (1756 from MEDLINE, 1093 from EMBASE, three from COCHRANE, and none from LILACS). After removing duplicates, 2841 articles were screened based on title and abstract, and 102 articles were evaluated using JBIQT. After quality evaluation, 33 articles were excluded, and 69 full-text articles were assessed for eligibility. Sixty articles met all the inclusion criteria. Figure 1 shows further detailed information regarding the inclusion of articles.

The search was updated on May 04, 2020, extracting 202 published articles (117 from MEDLINE, 85 from EMBASE, none from COCHRANE, and none from LILACS). After removing duplicates, 196 articles were screened based on title and abstract. A total of 11 articles were evaluated using JBIQT. After quality evaluation, two articles were discarded, and nine full-text articles were assessed for eligibility. Finally, after discarding two articles, as no ocular TEAEs were reported, seven articles met all the inclusion criteria (Fig. 1).

\section{Characteristics}

Our final inclusion strategy yielded 67 published articles, including 20 in the United States of America (USA), 24 in Europe (Germany, Spain, France, Italy, Wales, England, Switzerland, Croatia, and Sweden), three in Canada, three in Australia, nine in Asia (Japan, Israel, Iran, and Turkey), and eight were multicentric across continents.

All studies were published between 1987 and 2020 . Most of the studies included only adults, but one retrospective cross-sectional study included adults and children [8]. Of the 67 studies, 38 were case reports, four were case series, 14 were cross-sectional studies, and 11 were experimental studies. Table 1 summarizes the characteristics of the studies included in the present systematic review.

\section{Experimental studies}

A total of 11 experimental studies were included [9-19], which were published between 2010 and 2019. Six were multicentric across continents, three were conducted in the USA, one was conducted in Japan, and one was conducted in Iran.

After JBIQT qualification, the average number of items marked "Yes" across the studies was 11/13 (range 1013) for the RCTs, and one study was a nonrandomized controlled trial with $7 / 9$ of the items marked "Yes." Six studies had a sample size $\leq 400$ patients. 


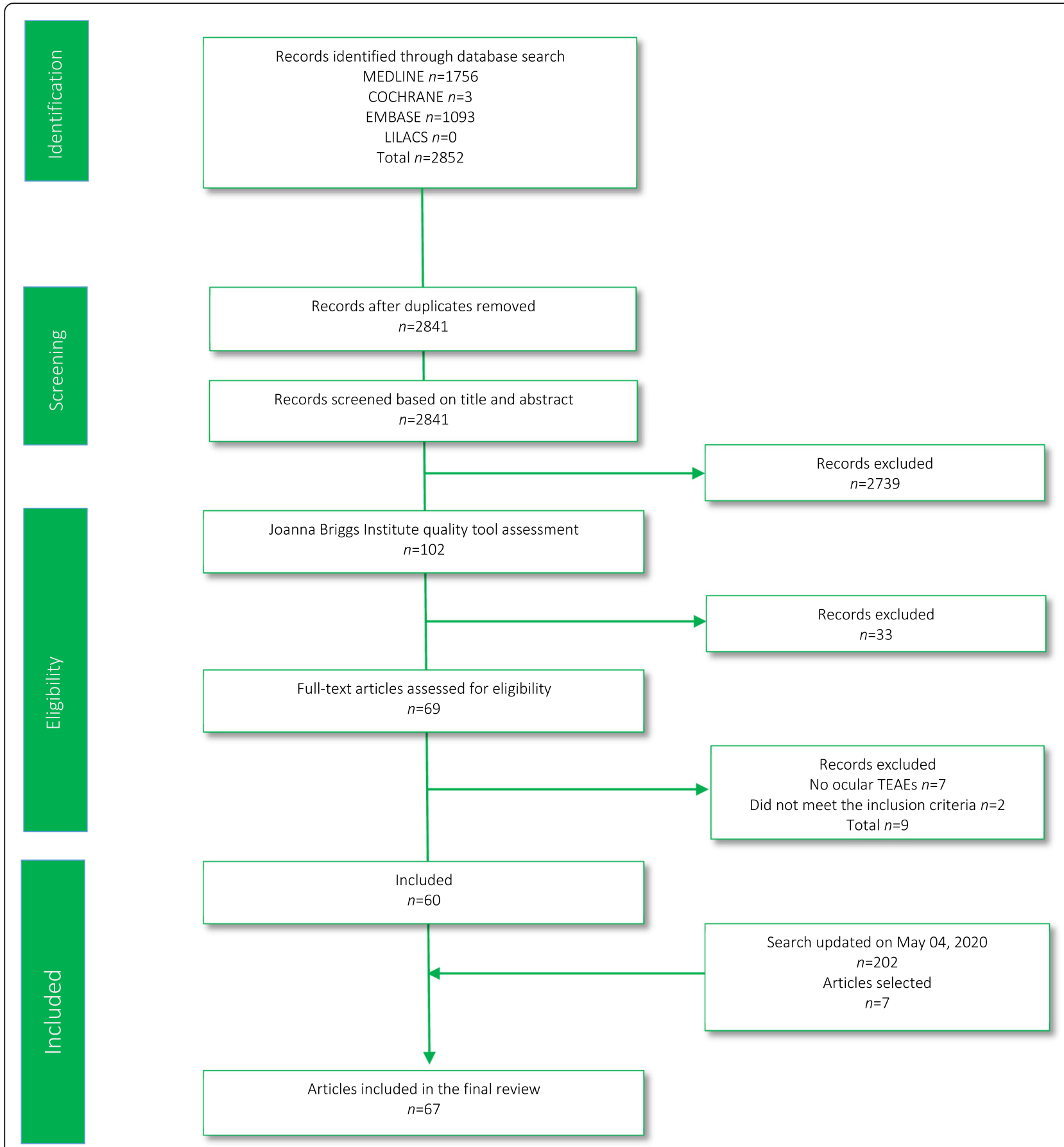

Fig. 1 PRISMA flowchart

\section{Observational studies}

We collected 56 observational studies, of which 38 were case reports [20-58], four were case series [59-61], and 14 were cross-sectional studies $[8,62-75]$. These studies were published between 1987 and 2020. After JBIQT qualification, the average number of items marked "Yes" across the observational studies was $7 / 8$ (range 6-8) for the cross-sectional studies, $7 / 8$ (range 6-8) for case reports, and 9/10 (range 6-10) for case series.

\section{Adverse events related to each therapy}

TEAEs related to alemtuzumab

Five studies from Germany, USA, and the United Kingdom (UK) referred to TEAEs when using alemtuzumab, 
Table 1 Characteristics of studies

\begin{tabular}{|c|c|c|c|c|c|c|c|}
\hline Author & Year & $\begin{array}{l}\text { Study } \\
\text { design }\end{array}$ & Country & Sex & $\begin{array}{l}\text { Patients with } \\
\text { TEAEs }\end{array}$ & TEAE & Post-TEAE \\
\hline \multicolumn{8}{|c|}{ ALEMTUZUMAB } \\
\hline $\begin{array}{l}\text { Tsourdi et al } \\
\text { [59] }\end{array}$ & 2015 & $\begin{array}{l}\text { Case } \\
\text { series }\end{array}$ & Germany & Both & $2 / 5$ & Thyroid eye disease & $\begin{array}{l}\text { Definitive treatment still discussed / Near-total } \\
\text { thyroidectomy and } 100 \mu \mathrm{g} \text { levothyroxine per } \\
\text { day }\end{array}$ \\
\hline $\begin{array}{l}\text { Trinh et al } \\
\text { [53] }\end{array}$ & 2015 & $\begin{array}{l}\text { Case } \\
\text { report }\end{array}$ & USA & Female & $1 / 1$ & Thyroid eye disease & $\begin{array}{l}\text { Improvement after thyroidectomy and } \\
\text { conservative management }\end{array}$ \\
\hline $\begin{array}{l}\text { Roos et al } \\
{[61]}\end{array}$ & 2018 & $\begin{array}{l}\text { Case } \\
\text { series }\end{array}$ & England & Both & $6 / 162$ & Thyroid eye disease & $\begin{array}{l}\text { Three patients required systemic } \\
\text { immunosuppression and three were } \\
\text { managed conservatively }\end{array}$ \\
\hline $\begin{array}{l}\text { Tuohy et al } \\
{[70]}\end{array}$ & 2014 & $\begin{array}{l}\text { Cross- }^{-} \\
\text {sectional }\end{array}$ & England & Both & $2 / 87$ & $\begin{array}{l}\text { Herpes zoster } \\
\text { ophthalmicus }\end{array}$ & Not reported \\
\hline $\begin{array}{l}\text { Willis et al } \\
\text { [72] }\end{array}$ & 2016 & $\begin{array}{l}\text { Cross- } \\
\text { sectional }\end{array}$ & $\begin{array}{l}\text { Wales and } \\
\text { England }\end{array}$ & Both & $1 / 100$ & Conjunctivitis & Not reported \\
\hline \multicolumn{8}{|l|}{ AMANTADINE } \\
\hline $\begin{array}{l}\text { Jeng et al } \\
{[35]}\end{array}$ & 2008 & $\begin{array}{l}\text { Case } \\
\text { report }\end{array}$ & USA & Both & $3 / 3$ & Corneal edema & $\begin{array}{l}\text { Improvement after treatment withdrawal in } \\
\text { two patients. One patient required } \\
\text { penetrating keratoplasty }\end{array}$ \\
\hline $\begin{array}{l}\text { Esquenazi } \\
{[30]}\end{array}$ & 2009 & $\begin{array}{l}\text { Case } \\
\text { report }\end{array}$ & USA & Female & $1 / 1$ & Corneal edema & $\begin{array}{l}\text { Improvement after treatment withdrawal and } \\
\text { topical prednisolone } 1 \%\end{array}$ \\
\hline \multicolumn{8}{|l|}{ FINGOLIMOD } \\
\hline $\begin{array}{l}\text { Calabresi } \\
\text { et al [9] }\end{array}$ & 2014 & $\mathrm{RCT}$ & Multicentric & Both & $9 / 1083$ & Macular edema & Improvement after treatment withdrawal \\
\hline $\begin{array}{l}\text { Cohen et al } \\
{[10]}\end{array}$ & 2010 & $\mathrm{RCT}$ & Multicentric & Both & $6 / 1292$ & Macular edema & Improvement after treatment withdrawal \\
\hline $\begin{array}{l}\text { Akiyama } \\
\text { et al [21] }\end{array}$ & 2016 & $\begin{array}{l}\text { Case } \\
\text { report }\end{array}$ & Japan & Female & $1 / 1$ & Macular edema & $\begin{array}{l}\text { Patient refused treatment withdrawal and } \\
\text { macular edema resolved }\end{array}$ \\
\hline $\begin{array}{l}\text { Chui et al } \\
{[27]}\end{array}$ & 2013 & $\begin{array}{l}\text { Case } \\
\text { report }\end{array}$ & Australia & Female & $1 / 1$ & Macular edema & $\begin{array}{l}\text { Patient refused treatment withdrawal and } \\
\text { improvement was observed after treatment } \\
\text { with ketorolac and dexamethasone }\end{array}$ \\
\hline $\begin{array}{l}\text { Jasani et al } \\
\text { [34] }\end{array}$ & 2017 & $\begin{array}{l}\text { Case } \\
\text { report }\end{array}$ & England & Female & $1 / 1$ & Macular edema & Improvement after treatment withdrawal \\
\hline Kim et al [37] & 2015 & $\begin{array}{l}\text { Case } \\
\text { report }\end{array}$ & USA & Female & $1 / 1$ & Macular edema & $\begin{array}{l}\text { Improvement after treatment withdrawal, } \\
\text { topical ketorolac and topical prednisolone }\end{array}$ \\
\hline $\begin{array}{l}\text { Minuk et al } \\
{[41]}\end{array}$ & 2013 & $\begin{array}{l}\text { Case } \\
\text { report }\end{array}$ & USA & Female & $1 / 1$ & Macular edema & $\begin{array}{l}\text { Patient refused treatment withdrawal and } \\
\text { improvement was observed after sub-tenon } \\
\text { triamcinolone injection }\end{array}$ \\
\hline $\begin{array}{l}\text { Schröder } \\
\text { et al [48] }\end{array}$ & 2015 & $\begin{array}{l}\text { Case } \\
\text { report }\end{array}$ & Germany & Female & $1 / 1$ & Macular edema & $\begin{array}{l}\text { Treatment was discontinued when ischemic } \\
\text { findings appeared }\end{array}$ \\
\hline $\begin{array}{l}\text { Thoo et al } \\
{[52]}\end{array}$ & 2014 & $\begin{array}{l}\text { Case } \\
\text { report }\end{array}$ & Australia & Female & $2 / 2$ & Macular edema & $\begin{array}{l}\text { Patient refused treatment withdrawal and } \\
\text { improvement was observed after intravitreal } \\
\text { triamcinolone injection }\end{array}$ \\
\hline $\begin{array}{l}\text { Turaka et al } \\
{[54]}\end{array}$ & 2012 & $\begin{array}{l}\text { Case } \\
\text { report }\end{array}$ & USA & Male & $1 / 1$ & Macular edema & Improvement after treatment withdrawal \\
\hline $\begin{array}{l}\text { Cifuentes- } \\
\text { Canorea et al } \\
{[57]}\end{array}$ & 2019 & $\begin{array}{l}\text { Case } \\
\text { report }\end{array}$ & Spain & Female & $1 / 1$ & Macular edema & $\begin{array}{l}\text { Improvement after treatment withdrawal and } \\
\text { reappearance after restart }\end{array}$ \\
\hline $\begin{array}{l}\text { Husmann } \\
\text { et al [58] }\end{array}$ & 2020 & $\begin{array}{l}\text { Case } \\
\text { report }\end{array}$ & USA & Female & $1 / 1$ & Macular edema & $\begin{array}{l}\text { Treatment was not withdrawn. Macular } \\
\text { edema improved with topical nepafenac }\end{array}$ \\
\hline $\begin{array}{l}\text { Lapierre et al } \\
\text { [63] }\end{array}$ & 2016 & $\begin{array}{l}\text { Cross- } \\
\text { sectional }\end{array}$ & Canada & Both & 11 and 2/2399 & $\begin{array}{l}\text { Macular edema and } \\
\text { uveitis }\end{array}$ & Improvement after treatment withdrawal \\
\hline $\begin{array}{l}\text { Laroni et al } \\
\text { [64] }\end{array}$ & 2016 & $\begin{array}{l}\text { Cross- }^{-} \\
\text {sectional }\end{array}$ & Italy & Both & $3 / 825$ & Macular edema & $\begin{array}{l}\text { 2/3 patients withdrew treatment; one } \\
\text { remained with macular edema and one } \\
\text { improved. In the last patient, treatment was } \\
\text { temporarily interrupted and restarted after }\end{array}$ \\
\hline
\end{tabular}


Table 1 Characteristics of studies (Continued)

\begin{tabular}{|c|c|c|c|c|c|c|c|}
\hline Author & Year & $\begin{array}{l}\text { Study } \\
\text { design }\end{array}$ & Country & Sex & $\begin{array}{l}\text { Patients with } \\
\text { TEAEs }\end{array}$ & TEAE & Post-TEAE \\
\hline & & & & & & & improvement \\
\hline $\begin{array}{l}\text { Ontaneda } \\
\text { et al [67] }\end{array}$ & 2012 & $\begin{array}{l}\text { Cross- }^{-} \\
\text {sectional }\end{array}$ & USA & Both & $3 / 317$ & Macular edema & Undetermined \\
\hline $\begin{array}{l}\text { Afshar et al } \\
\text { [20] }\end{array}$ & 2013 & $\begin{array}{l}\text { Case } \\
\text { series }\end{array}$ & USA & Both & $3 / 3$ & $\begin{array}{l}\text { Cystoid macular } \\
\text { edema }\end{array}$ & $\begin{array}{l}\text { Improvement after treatment withdrawal was } \\
\text { seen in two patients. One patient continued } \\
\text { fingolimod, with improvement after treatment } \\
\text { with nepafenac and diflurprednate }\end{array}$ \\
\hline $\begin{array}{l}\text { Asensio- } \\
\text { Sánchez et al } \\
{[22]}\end{array}$ & 2014 & $\begin{array}{l}\text { Case } \\
\text { report }\end{array}$ & Spain & Female & $1 / 1$ & $\begin{array}{l}\text { Cystoid macular } \\
\text { edema }\end{array}$ & No improvement after treatment withdrawal \\
\hline $\begin{array}{l}\text { Fan Gaskin } \\
\text { et al [33] }\end{array}$ & 2015 & $\begin{array}{l}\text { Case } \\
\text { report }\end{array}$ & Australia & Female & $1 / 1$ & $\begin{array}{l}\text { Cystoid macular } \\
\text { edema }\end{array}$ & $\begin{array}{l}\text { Improvement after treatment withdrawal, } \\
\text { topical diclofenac and topical prednisolone }\end{array}$ \\
\hline Pul et al [44] & 2016 & $\begin{array}{l}\text { Case } \\
\text { report }\end{array}$ & Germany & Female & $1 / 1$ & $\begin{array}{l}\text { Cystoid macular } \\
\text { edema }\end{array}$ & $\begin{array}{l}\text { Improvement after treatment withdrawal and } \\
\text { intravitreal ranibizumab injection }\end{array}$ \\
\hline $\begin{array}{l}\text { Ueda et al } \\
\text { [55] }\end{array}$ & 2015 & $\begin{array}{l}\text { Case } \\
\text { report }\end{array}$ & Japan & Male & $1 / 1$ & $\begin{array}{l}\text { Cystoid macular } \\
\text { edema and retinal } \\
\text { hemorrhages }\end{array}$ & $\begin{array}{l}\text { Macular edema was treated with } \\
\text { betamethasone after a 13-week persistence } \\
\text { and hemorrhages resolved after } 24 \text { weeks }\end{array}$ \\
\hline $\begin{array}{l}\text { Zarbin et al } \\
\text { [73] }\end{array}$ & 2013 & $\begin{array}{l}\text { Cross- } \\
\text { sectional }\end{array}$ & Multicentric & Both & 19 and $1 / 2615$ & $\begin{array}{l}\text { Macular edema and } \\
\text { retinal branch vein } \\
\text { occlusion }\end{array}$ & Improvement after treatment withdrawal \\
\hline $\begin{array}{l}\text { Bhatti et al } \\
\text { [24] }\end{array}$ & 2013 & $\begin{array}{l}\text { Case } \\
\text { report }\end{array}$ & USA & Female & $1 / 1$ & Macular hemorrhage & Improvement after treatment withdrawal \\
\hline $\begin{array}{l}\text { Christopher } \\
\text { et al [26] }\end{array}$ & 2017 & $\begin{array}{l}\text { Case } \\
\text { report }\end{array}$ & USA & Female & $1 / 1$ & Conjuctival lymphoma & $\begin{array}{l}\text { Improvement after treatment withdrawal and } \\
\text { rituximab treatment }\end{array}$ \\
\hline $\begin{array}{l}\text { Gallego- } \\
\text { Pinazo et al } \\
{[32]}\end{array}$ & 2011 & $\begin{array}{l}\text { Case } \\
\text { report }\end{array}$ & Spain & Female & $1 / 1$ & $\begin{array}{l}\text { Retinal branch vein } \\
\text { occlusion }\end{array}$ & $\begin{array}{l}\text { Improvement after treatment withdrawal and } \\
\text { intravitreal ranibizumab injection }\end{array}$ \\
\hline Lim et al [76] & 2019 & $\begin{array}{l}\text { Cross- } \\
\text { Sectional }\end{array}$ & Multicentric & Both & $27 / 27,528$ & $\begin{array}{l}\text { Uveitis complicated } \\
\text { with macular edema }\end{array}$ & $\begin{array}{l}\text { One case required glaucoma topical } \\
\text { treatment, two cases oral prednisone and the } \\
\text { remaining cases received topical steroid } \\
\text { preparations }\end{array}$ \\
\hline \multicolumn{8}{|l|}{ STEROIDS } \\
\hline $\begin{array}{l}\text { Saatci et al } \\
{[45]}\end{array}$ & 2010 & $\begin{array}{l}\text { Case } \\
\text { report }\end{array}$ & Turkey & Male & $1 / 1$ & Acute retinal necrosis & $\begin{array}{l}\text { No improvement after treatment withdrawal. } \\
\text { Vitrectomy and photocoagulation were } \\
\text { required, and retinal detachment occurred } \\
\text { after } 2 \text { months }\end{array}$ \\
\hline $\begin{array}{l}\text { Sheikh et al } \\
\text { [49] }\end{array}$ & 2016 & $\begin{array}{l}\text { Case } \\
\text { report }\end{array}$ & USA & Female & $1 / 1$ & Acute retinal necrosis & No improvement after treatment withdrawal \\
\hline \multicolumn{8}{|l|}{ CTLA4lg } \\
\hline $\begin{array}{l}\text { Viglietta et al } \\
\text { [17] }\end{array}$ & 2008 & Non-RCT & USA & Both & $1 / 20$ & Visual field defect & Not reported \\
\hline \multicolumn{8}{|l|}{ ESTRIOL } \\
\hline $\begin{array}{l}\text { Voskuhl et al } \\
{[16]}\end{array}$ & 2016 & $\mathrm{RCT}$ & USA & Both & $4 / 158$ & $\begin{array}{l}\text { Visual defects (blurred } \\
\text { vision or diplopia) }\end{array}$ & Not reported \\
\hline \multicolumn{8}{|l|}{ INTERFERON- $\beta$} \\
\hline $\begin{array}{l}\text { Bakri et al } \\
{[23]}\end{array}$ & 2015 & $\begin{array}{l}\text { Case } \\
\text { report }\end{array}$ & USA & Female & $1 / 1$ & $\begin{array}{l}\text { Intraretinal } \\
\text { hemorrhages }\end{array}$ & $\begin{array}{l}\text { Treatment was continued under strict follow- } \\
\text { up as the patient was asymptomatic }\end{array}$ \\
\hline $\begin{array}{l}\text { Massougnes } \\
\text { et al [28] }\end{array}$ & 2016 & $\begin{array}{l}\text { Case } \\
\text { report }\end{array}$ & Switzerland & Male & $2 / 2$ & $\begin{array}{l}\text { Retina peripheral } \\
\text { bilateral } \\
\text { telangiectasiae }\end{array}$ & $\begin{array}{l}\text { Treatment was not withdrawn, and no } \\
\text { progression was evidenced }\end{array}$ \\
\hline $\begin{array}{l}\text { De Santi } \\
\text { et al [29] }\end{array}$ & 2005 & $\begin{array}{l}\text { Case } \\
\text { report }\end{array}$ & Italy & Female & $1 / 1$ & Sicca syndrome & $\begin{array}{l}\text { Treatment was not withdrawn and } \\
\text { improvement was observed with oral } \\
\text { methylprednisolone treatment }\end{array}$ \\
\hline Gaetani et al & 2015 & Case & Italy & Female & $1 / 1$ & Retinopathy & Improvement after treatment withdrawal \\
\hline
\end{tabular}


Table 1 Characteristics of studies (Continued)

\begin{tabular}{|c|c|c|c|c|c|c|c|}
\hline Author & Year & $\begin{array}{l}\text { Study } \\
\text { design }\end{array}$ & Country & Sex & $\begin{array}{l}\text { Patients with } \\
\text { TEAEs }\end{array}$ & TEAE & Post-TEAE \\
\hline [31] & & report & & & & & \\
\hline $\begin{array}{l}\text { Jenisch et al } \\
\text { [36] }\end{array}$ & 2012 & $\begin{array}{l}\text { Case } \\
\text { report }\end{array}$ & Germany & Female & $1 / 1$ & $\begin{array}{l}\text { Branch arterial } \\
\text { occlusions and central } \\
\text { vein occlusion }\end{array}$ & $\begin{array}{l}\text { Treatment was not withdrawn. Visual acuity } \\
\text { improvement was observed after } \\
\text { hydroxyethyl- starch and salicylic acid } \\
\text { treatment }\end{array}$ \\
\hline $\begin{array}{l}\text { Longmuir } \\
\text { et al [39] }\end{array}$ & 2007 & $\begin{array}{l}\text { Case } \\
\text { report }\end{array}$ & USA & Male & $1 / 1$ & Retinopathy & $\begin{array}{l}\text { Improvement after treatment temporal } \\
\text { suspension }\end{array}$ \\
\hline $\begin{array}{l}\text { Mallada- } \\
\text { Frechín et al } \\
{[40]}\end{array}$ & 2005 & $\begin{array}{l}\text { Case } \\
\text { report }\end{array}$ & Spain & Female & $1 / 1$ & Retinopathy & Improvement after treatment withdrawal \\
\hline $\begin{array}{l}\text { Ohira et al } \\
\text { [42] }\end{array}$ & 2009 & $\begin{array}{l}\text { Case } \\
\text { report }\end{array}$ & Japan & Female & $1 / 1$ & Retinopathy & Improvement after treatment withdrawal \\
\hline $\begin{array}{l}\text { Post et al } \\
{[43]}\end{array}$ & 2009 & $\begin{array}{l}\text { Case } \\
\text { report }\end{array}$ & Canada & Female & $1 / 1$ & $\begin{array}{l}\text { Visual field } \\
\text { disturbance }\end{array}$ & Improvement after treatment withdrawal \\
\hline $\begin{array}{l}\text { Saito et al } \\
\text { [46] }\end{array}$ & 2007 & $\begin{array}{l}\text { Case } \\
\text { report }\end{array}$ & Japan & Male & $1 / 1$ & Retinopathy & Improvement after treatment withdrawal \\
\hline $\begin{array}{l}\text { Sallansonnet- } \\
\text { Froment } \\
\text { et al [47] }\end{array}$ & 2009 & $\begin{array}{l}\text { Case } \\
\text { report }\end{array}$ & France & Male & $1 / 1$ & Retinopathy & Improvement after treatment withdrawal \\
\hline $\begin{array}{l}\text { Sommer } \\
\text { et al [50] }\end{array}$ & 2001 & $\begin{array}{l}\text { Case } \\
\text { report }\end{array}$ & France & Male & $1 / 1$ & Retinopathy & Improvement after treatment withdrawal \\
\hline $\begin{array}{l}\text { Spierer et al } \\
{[51]}\end{array}$ & 2011 & $\begin{array}{l}\text { Case } \\
\text { report }\end{array}$ & Israel & Female & $1 / 1$ & $\begin{array}{l}\text { Idiopathic orbital } \\
\text { inflammation }\end{array}$ & $\begin{array}{l}\text { Treatment was not withdrawn. Symptoms } \\
\text { improved with oral prednisolone }\end{array}$ \\
\hline $\begin{array}{l}\text { Williams et al } \\
\text { [60] }\end{array}$ & 2004 & $\begin{array}{l}\text { Case } \\
\text { series }\end{array}$ & USA & Both & $3 / 3$ & Neuroretinitis & Not reported \\
\hline $\begin{array}{l}\text { Makioka et al } \\
{[66]}\end{array}$ & 2017 & $\begin{array}{l}\text { Cross- } \\
\text { sectional }\end{array}$ & Japan & Both & $1 / 1476$ & Retinopathy & No improvement after treatment withdrawal \\
\hline $\begin{array}{l}\text { Smith et al } \\
\text { [69] }\end{array}$ & 2015 & $\begin{array}{l}\text { Cross- }^{-} \\
\text {sectional }\end{array}$ & USA & Both & $\begin{array}{l}\text { Incidence rate of } \\
4.04 / 100 \text { patients } \\
\text { per year of } 8107 \\
\text { adults }\end{array}$ & $\begin{array}{l}\text { Abnormal vision and } \\
\text { xerophthalmia }\end{array}$ & Undetermined \\
\hline $\begin{array}{l}\text { Tremlett } \\
\text { et al [8] }\end{array}$ & 2008 & $\begin{array}{l}\text { Cross- } \\
\text { sectional }\end{array}$ & Canada & Both & $1 / 888^{* *}$ & Papilledema & Not reported \\
\hline $\begin{array}{l}\text { Liscić et al } \\
\text { [65] }\end{array}$ & 2004 & $\begin{array}{l}\text { Cross- }^{-} \\
\text {sectional }\end{array}$ & Croatia & Both & $2 / 9$ & $\begin{array}{l}\text { Abnormal visual } \\
\text { evoked potentials }\end{array}$ & No improvement after treatment withdrawal \\
\hline $\begin{array}{l}\text { Gugliandolo } \\
\text { et al [74] }\end{array}$ & 2018 & $\begin{array}{l}\text { Cross- }^{-} \\
\text {sectional }\end{array}$ & $\begin{array}{l}\text { Italy (three } \\
\text { regions: } \\
\text { Liguria, Sicily, } \\
\text { and Sardinia) }\end{array}$ & Both & $2 / 6039$ & $\begin{array}{l}\text { Eyelid edema and } \\
\text { visual field defect }\end{array}$ & Visual field defect resolved within a day \\
\hline $\begin{array}{l}\text { Pakdaman } \\
\text { et al [18] }\end{array}$ & 2018 & $\mathrm{RCT}$ & Iran & Both & $\begin{array}{l}\text { Avonex: 3/89 } \\
\text { Cinnovez: 1/93 }\end{array}$ & Visual disturbance & Not reported \\
\hline $\begin{array}{l}\text { Comi et al } \\
{[77]}\end{array}$ & 2019 & $\mathrm{RCT}$ & Multicentric & Both & $\begin{array}{l}\text { Ozanimod } 1 \text { mg: } \\
\text { 1/447 } \\
\text { Ozanimod } 0.5 \text { mg: } \\
\text { 1/451 } \\
\text { Interferon-B1a: 1/ } \\
448\end{array}$ & Macular edema & Not reported \\
\hline \multicolumn{8}{|l|}{ NATALIZUMAB } \\
\hline $\begin{array}{l}\text { Boster et al } \\
\text { [25] }\end{array}$ & 2013 & $\begin{array}{l}\text { Case } \\
\text { report }\end{array}$ & USA & Male & $1 / 1$ & $\begin{array}{l}\text { Progressive Multifocal } \\
\text { Leukoencephalopathy } \\
\text { by JCV }\end{array}$ & Patient passed away \\
\hline $\begin{array}{l}\text { Zecca et al } \\
{[56]}\end{array}$ & 2009 & $\begin{array}{l}\text { Case } \\
\text { report }\end{array}$ & Switzerland & Male & $1 / 1$ & $\begin{array}{l}\text { Ocular toxoplasmosis } \\
\text { reactivation }\end{array}$ & $\begin{array}{l}\text { Treatment withdrawal and treatment with } \\
\text { pyrimethamine sulfadiazine, prednisolone and } \\
\text { folinic acid with lesion reduction }\end{array}$ \\
\hline $\begin{array}{l}\text { Holmén et al } \\
\text { [62] }\end{array}$ & 2011 & $\begin{array}{l}\text { Cross- } \\
\text { sectional }\end{array}$ & Sweden & Both & $19 / 85$ & Ocular inflammation & Not reported \\
\hline
\end{tabular}


Table 1 Characteristics of studies (Continued)

\begin{tabular}{|c|c|c|c|c|c|c|c|}
\hline Author & Year & $\begin{array}{l}\text { Study } \\
\text { design }\end{array}$ & Country & Sex & $\begin{array}{l}\text { Patients with } \\
\text { TEAEs }\end{array}$ & TEAE & Post-TEAE \\
\hline $\begin{array}{l}\text { Saida et al } \\
\text { [13] }\end{array}$ & 2016 & $\mathrm{RCT}$ & Japan & Both & $1 / 12$ & Retinal detachment & Undetermined \\
\hline \multicolumn{8}{|c|}{ HYPERBARIC OXYGEN } \\
\hline $\begin{array}{l}\text { Lambrou } \\
\text { et al [38] }\end{array}$ & 1987 & $\begin{array}{l}\text { Case } \\
\text { report }\end{array}$ & France & Female & $1 / 1$ & $\begin{array}{l}\text { Slow-recovering } \\
\text { central bilateral } \\
\text { scotoma }\end{array}$ & Improvement after treatment withdrawal \\
\hline \multicolumn{8}{|l|}{ RITUXIMAB } \\
\hline $\begin{array}{l}\text { Rommer } \\
\text { et al [68] }\end{array}$ & 2015 & $\begin{array}{l}\text { Cross- } \\
\text { sectional }\end{array}$ & Germany & Both & $1 / 56$ & Ocular inflammation & Not reported \\
\hline \multicolumn{8}{|l|}{ SIPONIMOD } \\
\hline $\begin{array}{l}\text { Selmaj et al } \\
{[14]}\end{array}$ & 2013 & $\mathrm{RCT}$ & Multicentric & Both & $1 / 297$ & $\begin{array}{l}\text { Macular edema and } \\
\text { optic neuritis }\end{array}$ & Undetermined \\
\hline $\begin{array}{l}\text { Kappos et al } \\
{[12]}\end{array}$ & 2018 & $\mathrm{RCT}$ & Multicentric & Both & 18/1099 & Macular edema & Not reported \\
\hline \multicolumn{8}{|c|}{ TERIFLUNOMIDE } \\
\hline $\begin{array}{l}\text { Vermersch } \\
\text { et al [15] }\end{array}$ & 2013 & $\mathrm{RCT}$ & Multicentric & Both & $\begin{array}{l}\text { Teriflunomide } 7 \\
\text { mg: } 3 / 111\end{array}$ & $\begin{array}{l}\text { Optic neuritis, macular } \\
\text { edema, and optic } \\
\text { ischemic neuropathy }\end{array}$ & Not reported \\
\hline \multicolumn{8}{|l|}{ TOVAXIN } \\
\hline Fox et al [11] & 2012 & $\mathrm{RCT}$ & USA & Both & $1 / 100$ & Diplopia & Not reported \\
\hline
\end{tabular}

** This study measured TEAEs, not patients

of which three reported the development of thyroid eye disease (TED) [53, 59, 61].

One of the studies was a case series extracted from two clinical trials (CARE-MS1 and CARE-MS2) where five patients developed Graves' disease (GD), but only two patients had TED [59]. According to Trinh et al [53], there was a female patient who developed bilateral ocular hyperemia, photophobia, proptosis, and upper eyelid retraction, consistent with TED, after three years of treatment [53]. A similar study from the UK reported 10 patients of 162 with GD, but only six patients presented TED, with a period of 26 months, 20 months, four years, 10 months, four years, and 48 months after the last infusion of alemtuzumab, respectively [61].

In a cross-sectional study conducted by Tuohy et al, thyroid autoimmunity developed in 35/87 patients (40.2\%) of whom 22/35 (63\%) had hyperthyroidism. Although the study reported GD, it was not clear whether the patients had any ophthalmological manifestation. Two patients in that study developed Herpes zoster ophthalmicus [70]. In another study, the use of alemtuzumab was related to thyroid gland autoimmunity (35\%), but no TED was reported, and $1 \%$ of the patients presented conjunctivitis; however, the study did not specify the etiology of conjunctival inflammation [72].

\section{TEAEs related to amantadine}

We found two case reports from the USA that described four cases of bilateral corneal edema as a TEAE related to amantadine used for managing tremor and fatigue in patients with MS [30,35]. The duration of amantadine treatment before the onset of symptoms in all cases ranged from two months to six years, and the range of corneal thickening was $677 \mu \mathrm{m}$ to $>1000 \mu \mathrm{m}$ [30, 35]. In two cases, the corneal edema resolved approximately one month after amantadine was suspended [30, 35]; in one case, the corneal edema resolved two months after amantadine suspension [35]; and in one case, the corneal edema required management with bilateral penetrating keratoplasty [35].

\section{TEAEs related to fingolimod}

A total of 25 studies reported ocular TEAEs in patients treated with fingolimod. Of these studies, 18 were case reports, mostly from the USA, Australia, Spain, and Germany; two were experimental studies, both multicentric; and five were cross-sectional studies, one from Canada, one from Italy, one from the USA, and two were multicentric.

Macular edema was reported in 21 studies [9, 10, 20$22,27,33,34,37,41,44,48,52,54,55,57,58,63,64$, $67,73]$; five were classified as cystoid macular edema $[20,22,33,44]$, and one study reported associated retinal hemorrhages [55]. One study described that the symptoms entirely resolved after the discontinuation of medication; when the medication was restarted after two months, the symptomatology reappeared [57]. One patient had macular edema and retinal branch vein 
occlusion [73], and one patient had macular hemorrhage [24]. In one case report, fingolimod was not withdrawn, and topical nepafenac was used, which improved the macular edema [58]. Of the remaining three studies, one [26] reported conjunctival lymphoma, one reported retinal branch vein occlusion [32], and one described uveitis complicated with macular edema. In the last study, the authors inferred that the TEAE could not be exclusively attributed to fingolimod because uveitis can by itself cause macular edema [76].

\section{TEAEs related to steroids}

We found two case reports that described acute retinal necrosis as a TEAE after high-dose steroid treatment for relapsing-remitting multiple sclerosis (RRMS), one from Turkey and the other from the USA $[45,49]$. In the first report, the patient received an IV steroid pulse of 1000 $\mathrm{mg} /$ day for three days and was continued with $10 \mathrm{mg}$ of daily prednisolone. After two months, the visual symptoms started and the diagnosis of acute retinal necrosis caused by the Varicella-zoster virus was made [45]. In the other case report, the patient received three courses of high-dose methylprednisolone $(1000 \mathrm{mg} /$ day for three days) for recurrent relapses five months before the presentation of visual symptoms. The diagnosis of Herpes simplex virus type 2 infection was made [49].

\section{TEAEs related to CTLA-4 Ig}

This immunoglobulin was used in a Phase 1 clinical trial conducted on 20 patients with RRMS from the USA, which evaluated its safety and tolerability in different doses. This study reported, a visual field defect in one patient receiving a $2 \mathrm{mg} / \mathrm{kg}$ dose of the drug within the first $24 \mathrm{~h}$ of infusion. During the long-term follow-up, $10 \%$ of the enrolled patients had blurred vision [17].

\section{TEAEs related to estriol}

A Phase 2 clinical trial conducted on patients with RRMS from 16 academic neurology clinics in the USA, evaluating the effect of estriol treatment in the reduction of MS relapses in women, reported TEAEs within 24 months of treatment. There were six events of visual defects (blurred vision or diplopia) reported in four patients receiving estriol compared to seven of the same visual defects reported in seven placebo patients [16].

\section{TEAEs related to interferon $\beta$}

Our review included 21 studies that involved TEAEs after INF therapy, mostly in patients with RRMS.

There were 13 case reports, mostly from Europe $(n=$ $7)$, USA $(n=2)$, Japan $(n=2)$, Canada $(n=1)$, and Israel $(n=1)$. Most of the patients were women $(n=8)$. Patients' age ranged from 30 to 58 years. The majority of them complained about symptoms after 11 months since the initiation of the treatment. The TEAEs reported in these patients included unilateral/bilateral asymptomatic retinopathy (peripheral intraretinal hemorrhages and cotton wool spots), unilateral/bilateral symptomatic retinopathy (blurred vision, progressive visual field loss, and peripheral telangiectasia), central vein and arterial branch occlusions, Sicca syndrome, and recurrent idiopathic orbital inflammation $[23,28,29,31,36,39,40$, $42,43,46,47,50,51]$.

One study was a case series that reported three patients with neuroretinitis after INF $\beta$ therapy, of whom two had been undergoing INF therapy for around a year before the symptoms appeared, the other patient presented with a macular star exudate on funduscopic examination concurrently with the initiation of treatment [60].

There were five cross-sectional studies (one each from Japan, the USA, Canada, Croatia, and Italy). Makioka et al. reported only one case of retinopathy in a postmarketing drug surveillance study for INF $\beta-1 \mathrm{a}$ intramuscular injection in Japan, among a sample of 1441 patients [66]. Smith et al. conducted a postmarketing safety profile study in adult patients with MS with a prescription of INF $\beta$-1a SC three times a week between 2006 and 2012 . Some of the patients complained about abnormal vision and xerophthalmia. There were reports of retinal artery or vein obstructions and retinopathy [69]. Tremlett et al. summarized the reported TEAEs for 10 years in Canada in the adult and children population. A 43-year-old female patient presented with papilledema and visual disturbance related to malignant neoplasm aggravation and resulted in death [8]. Liscić et al. evaluated patternreversal visual evoked potentials (VEP) in patients with RRMS on INF $\beta$ - 1 a treatment ( $n=9$ patients, 18 eyes). Those with previous optic neuritis $(n=3$ patients, 3 eyes) exhibited visual evoked potential impairment with a delay in P100 latency before treatment. Moreover, some patients ( $n=2$ patients, 3 eyes) without previous impairment exhibited increased P100 latency after INF therapy. It is not clear whether this impairment is due to INF therapy or MS itself [65]. A multicentric pharmacovigilance study conducted to analyze 10 drugs used for MS treatment over a period of 24 months recorded 411 adverse reactions. Approximately $42 \%$ of the TEAEs were due to INF, from which approximately $9 \%$ were unexpected, and $5.8 \%$ were severe events. Two patients presented ophthalmological manifestations. The first patient, associated with peginterferon- $\beta-1 \mathrm{a}$, with previous use of dimethyl fumarate, presented mild-moderate eyelid edema. The second patient, associated with glatiramer acetate, with previous use of INF $\beta-1 \mathrm{a}$, presented moderate-severe visual field defect, which resolved within a day [74].

Finally, we included two experimental studies. The first one was a double-blind RCT evaluating the 
comparative efficacy and safety of two different trademarks of INF $\beta-1 \mathrm{a}$ in patients with RRMS conducted by Pakdaman et al. It included 182 participants, of whom 89 received trademark A and 93 trademark B. Regarding TEAEs, 3.4\% of participants receiving trademark $\mathrm{A}$ and $1.1 \%$ receiving trademark $B$ reported visual disturbances [18]. The second study was a multicentric, randomized Phase 3 clinical trial conducted by Comi et al. The aim was to evaluate the safety and efficacy of ozanimod compared with INF $\beta$-1a in patients with RRMS. It enrolled 1346 participants, of whom 447 received $1 \mathrm{mg}$ of ozanimod, 451 received $0.5 \mathrm{mg}$ of ozanimod, and 448 received INF $\beta$-1a. One participant in each treatment group reported a TEAE. The patient in the INF $\beta$-1a group presented macular edema [77].

\section{TEAEs related to natalizumab}

We found four articles regarding natalizumab [13, 25, $56,62]$, including two case reports (one from the USA and the other from Switzerland), one cross-sectional study (from Sweden), and one RCT (from Japan).

Of the two case reports, one [25] reported a case of progressive multifocal leukoencephalopathy (PML) associated with John Cunningham virus (JVC) that started with a subacute onset of bilateral blindness after the 44th dose of natalizumab. The second study reported the first published case of ocular toxoplasmosis reactivation during natalizumab treatment [56].

A web-based MS registry cross-sectional Swedish national postmarketing surveillance study reported 19 patients with TEAEs related to ocular inflammation, herpes simplex, herpes zoster, urinary tract infection, enterovirus meningitis, increased infection susceptibility, and hepatitis $\mathrm{C}$ infection. However, the study did not clarify how many of the 19 patients presented the eye compromise. Furthermore, the study reported three cases of PML that occurred during these trial periods, including two cases in the open-label extension phase of the SENTINEL trial and one case in a clinical trial for Crohn's disease [78, 79]. There was no information regarding the visual compromise in the follow-up of patients who developed PML [62].

The RCT reported a patient with retinal detachment $75 \mathrm{~d}$ after the first dose of natalizumab [13].

\section{TEAEs related to hyperbaric oxygen}

Of the 62 included articles, only one case report published in 1986 described TEAE due to hyperbaric oxygen in a patient with MS who presented slow-recovering central bilateral scotoma [63].

\section{TEAEs related to rituximab}

One case of ocular inflammation as a TEAE was found after the initiation of treatment, which was reported at the German Registry of autoimmune diseases (GRAID) in a multicentric retrospective study. The time between treatment onset and the TEAE was not determined [68].

\section{TEAEs related to siponimod}

We found two studies regarding siponimod. One was a multicentric Phase 2 clinical trial conducted on patients with RRMS from 73 specialized MS centers in Canada, the USA, Russia, and nine European countries, wherein the patients were divided into cohort $1(n=188)$ and cohort $2(n=109)$. It reported that one patient with a history of uveitis presented macular edema with the highest dose of the drug $(10 \mathrm{mg})$, and one patient presented optic neuritis with $0.5-\mathrm{mg}$ dose of the drug [14]. The second study was a Phase 3 clinical trial conducted on patients with secondary progressive multiple sclerosis (SPMS) from 292 hospitals, clinics, and specialized MS centers in 31 countries. It reported that $2 \%(n=18)$ of patients receiving siponimod presented macular edema compared to $<1 \%(n=1)$ of those receiving placebo [12].

\section{TEAEs related to teriflunomide}

In an experimental study conducted on patients with RRMS from different countries of America, Europe, and Africa, 324 patients were divided into the following three treatment groups: INF $\beta-1 \mathrm{a}(n=104)$, teriflunomide 7 $\mathrm{mg}(n=109)$, and teriflunomide $14 \mathrm{mg}(n=111)$. The second group was treated for 66.6 weeks, and the third group was treated for 64.2 weeks. The ocular TEAEs reported were optic neuritis, macular edema, and ischemic optic neuropathy in the second group of patients who received $7 \mathrm{mg}$ of teriflunomide. No ocular TEAEs were observed in groups 1 and 3 [15].

\section{TEAEs related to tovaxin}

We found an experimental study that evaluated the safety of tovaxin for 24 weeks in patients with RRMS from 30 to 40 different sites in the USA. Neither deaths nor discontinuations due to safety reasons were reported in that study. Diplopia was the only reported ocular event, and it was considered as a major reaction because of the necessity of in-hospital attention of the patient, but not because of the event itself. The authors did not consider this reaction as secondary to the drug and evaluated tovaxin as a safe treatment for the course of 24 weeks [11].

\section{Discussion}

Methodological assessment of TEAEs

In 2016, PRISMA published an article about harmreporting in systematic reviews, where terms such as adverse drug reaction, adverse effect, adverse event, complication, harm, side effect, and toxicity were discussed [80]. However, there exists a large heterogeneity when 
using these terms and reporting TEAEs. This represents a difficulty at the time of reading and interpreting articles about drug safety. Moreover, the diversity between the different analytic and data collection strategies, the different forms of presentation of TEAEs, and the variety among the study designs represented additional challenges in this systematic review.

Two available strategies should be used to record information about TEAEs, i.e., active monitoring, when an event is known or suspected to be associated with an intervention, and spontaneous monitoring for new or unexpected events [81]. Different methodological designs were included in this systematic review because active monitoring generally evaluates long-term TEAEs in RCTs and short-term TEAEs in large cohort studies or case-control studies [81], whereas spontaneous monitoring commonly describes TEAEs in case series and case reports. Each study included in this systematic review had to be carefully evaluated, because spontaneous reports may lead to underreporting and false positives, RCTs may not identify new events, observational studies may require a large sample size, and case reports may not be well documented and have a long-time elapse until publication [82].

\section{Geographical distribution}

Developed countries have a higher incidence of MS. As the populations in these countries have better access to healthcare and early treatment strategies, it is expected that most of the studies that evaluated adverse ophthalmological events - and adverse events in general - had been conducted in these countries. This is consistent with our results, which indicated that studies were largely conducted in Europe, North America, and Japan. A few case reports were described in Australia and Israel. Some multicentric studies included a couple of

Table 2 Referral recommendations for each TEAE

\begin{tabular}{|c|c|}
\hline TEAEs & Referral \\
\hline Papilledema & Neurologist, priority attention \\
\hline Conjunctivitis & Ophthalmologist \\
\hline Herpes zoster ophtalmicus & Ophthalmologist and refer depending on the compromise \\
\hline \multicolumn{2}{|l|}{ Visual disturbance } \\
\hline \multicolumn{2}{|l|}{ Ocular inflammation } \\
\hline Macular edema & Ophthalmologist, if available retina specialist \\
\hline \multicolumn{2}{|l|}{ Retinal branch vein occlusion } \\
\hline \multicolumn{2}{|l|}{ Macular hemorrhage } \\
\hline \multicolumn{2}{|c|}{ Retinal hemorrhages and macular edema } \\
\hline \multicolumn{2}{|l|}{ Retinopathy } \\
\hline \multicolumn{2}{|l|}{ Intraretinal hemorrhages } \\
\hline \multicolumn{2}{|c|}{ Retina peripheral bilateral telangiectasiae } \\
\hline \multicolumn{2}{|l|}{ Neuroretinitis } \\
\hline \multicolumn{2}{|l|}{ Retinal detachment } \\
\hline \multicolumn{2}{|l|}{ Acute retinal necrosis } \\
\hline Ocular toxoplasmosis reactivation & Ophthalmologist, if available retina or uvea specialist \\
\hline Uveitis & Ophthalmologist, if available uvea specialist \\
\hline Abnormal visual evoked potentials & Ophthalmologist, if available neuro-ophthalmologist \\
\hline \multicolumn{2}{|l|}{ Visual field defect } \\
\hline \multicolumn{2}{|c|}{ Progressive multifocal leukoencephalopathy by JCV } \\
\hline \multicolumn{2}{|l|}{ Optic ischemic neuropathy } \\
\hline \multicolumn{2}{|l|}{ Diplopia } \\
\hline Thyroid eye disease & Endocrinologist and ophthalmologist, if available oculoplastics specialist \\
\hline Eyelid edema & Ophthalmologist, if available oculoplastics specialist \\
\hline \multicolumn{2}{|l|}{ Conjuctival lymphoma } \\
\hline Idiopathic orbital inflammation & Ophthalmologist, if available ocular surface specialist \\
\hline Sjogren syndrome & Rheumatologist and ophthalmologist, if available ocular surface specialist \\
\hline Corneal edema & Ophthalmologist, if available cornea specialist \\
\hline
\end{tabular}


Asian countries apart from Japan as well. There were no reports of adverse events from other countries or regions, which may result in a poor understanding of the entire picture worldwide.

\section{Pregnancy and hormones in MS}

MS relapses are decreased during pregnancy (primarily during the third trimester) and influenced by sex hormones such as estriol [16]. One RCT demonstrated that estriol was related to visual defects (blurred or double vision); nonetheless, the same visual defects were reported in the placebo group. In clinically isolated syndromes, the incidence of some of the typical features such as visual acuity and color vision was reduced [83]; therefore, the findings might not be directly related to estriolassociated TEAEs. These manifestations have also been described in acute optic neuritis, in which patients described blurred vision [84]. Furthermore, optic neuritis was related to different MS treatments such as INF $\beta-1 b$, siponimod, and teriflunomide [14, 15, 85]. However, the resolution of TEAEs after medication withdrawal was not described.

\section{Follow-up recommendations}

Optic neuritis is one of the primary manifestations of MS; therefore, ophthalmological follow-up is mandatory in these patients. Complete ophthalmological examination with attention to the efferent visual system and ancillary studies such as VEP and optic nerve optical coherence tomography (OCT) should be available for these patients [86].

Based on the present study, fingolimod-related TEAEs were highly common. We suggest the following American Academy of Ophthalmology recommendations for patients with MS being treated with fingolimod published in October 2011: 1) A screening evaluation for pre-existing uveitis or macular or retinal vascular disease before starting, or within the first few weeks of starting fingolimod. 2) A single re-evaluation at 3-4 months of therapy. 3) Patients should be advised that the incidence of macular edema is low $(\sim 2 / 1000)$, but if there is a history of uveitis, the incidence may be as high as $20 \%$. 4) Visual acuity check and a complete eye exam, including a dilated fundus exam, is a proper ophthalmic screening protocol. 5) Patients with abnormalities on the exam or unexplained decreased visual acuity might benefit from diagnostic imaging with macular OCT [87]. These recommendations are supported by the methodology used in the study conducted by Zarbin et al. in 2013, who reported differences in different times of evaluation of these patients [73].

Regarding the use of alemtuzumab in patients with MS and thyroid disease, it was reported that up to $22 \%$ of patients treated with alemtuzumab would develop GD
[53]. In almost $90 \%$ of the cases, GD and Graves ophthalmopathy (GO) co-occur; nevertheless, GO can develop after up to 1 year from being diagnosed with GD [88]. Therefore, we suggest an annual check-up by ophthalmologists, neurologists, and endocrinologists. This follow-up is important to ensure that patients undergo timely ophthalmological treatment while simultaneously ensuring the continued success of alemtuzumab treatment in the management of MS [53, 59, 70].

In contrast, one of the studies on patients treated with alemtuzumab reported Herpes zoster ophthalmicus infection [70]. Previous trials have identified Herpes simplex virus and Varicella-zoster virus systemic opportunistic infections are among the most common infections in patients treated with alemtuzumab and recommended preventive measures, including antiherpetic prophylaxis [89].

Studies evaluating the presence of corneal edema secondary to the use of amantadine in patients with MS corresponded to case reports. Considering that corneal findings can occur from two months to six years after medication initiation, we recommend that the neurologist treating the patient be aware of the warning signs to immediately consult the ophthalmology specialist and prevent irreversible endothelial failure [30, 35].

Another study evaluated the presence of dry eye after the use of anticholinergics, based on which we recommend that the neurologist treating patients with MS must be aware of this TEAE. Although the symptoms should not be the reason to stop an efficient treatment, it is recommended to conduct an ophthalmological evaluation to treat dry eye [71].

The study that evaluated CTLA-4 Ig treatment in patients with MS demonstrated blurred vision in $10 \%$ of the patients. However, the cause of blurred vision was not clear, and the spectrum of MS manifestations included blurred vision secondary to optic neuritis and uveitis. This is why further studies are required to evaluate the safety of this drug in patients with MS, assess whether the ocular compromise corresponds to a TEAE, and generate recommendations on this issue [17].

The clinical trial comparing estriol combined with glatiramer acetate and placebo for women with RRMS reported six blurry and double vision events after six months of follow-up. However, the study did not report the etiology of the visual problems, and hence it is not possible to know whether it corresponds to the natural course of the disease. Further studies are necessary to evaluate the safety of this medication [16].

Two case reports described acute retinal necrosis caused by HSV-2 and Varicella-zoster virus in patients undergoing high-dose steroid therapy $[45,49]$. Therefore, we recommend a retina specialist evaluation for patients showing ocular symptoms such as floaters, blurred 
vision, and sudden vision loss after treatment with steroid pulses.

Patients undergoing INF $\beta$-1a therapy showed multiple ocular TEAEs in a duration range of three months to 10 years (Table 1). Given the wide timeframe for the establishment of TEAEs, and that some of the pathologies may correspond to manifestations or associations of MS, we consider that the most appropriate recommendation for these patients is the evaluation by an ophthalmologist before starting treatment and at least once a year after the start of treatment.

We consider that there is insufficient information to provide screening recommendations regarding natalizumab, hyperbaric oxygen, rituximab, siponimod, teriflunomide, and tovaxin. Nevertheless, in the presence of any ocular symptoms, we strongly recommend an immediate evaluation by an ophthalmology specialist.

Table 2 shows the referral recommendations for each TEAE reported in this systematic review, based on our clinical experience. However, it is important to consider that each patient's case should be individualized, and disease guidelines should be followed.

\section{Conclusions}

Considering that ophthalmological TEAEs are generally undermined in clinical trials, different methodological designs were included in this systematic review. In light of this, it is necessary to conduct longitudinal studies to provide evidence-based recommendations.

Despite the diverse geographical distribution of the studies, there were scarce data from Latin America and African countries. Therefore, we strongly encourage healthcare providers to conduct pilot studies in these regions.

We suggest that physicians perform a comprehensive interrogatory and evaluation for ocular signs and symptoms during the follow-up of patients receiving treatment for MS and refer when necessary. An interdisciplinary approach might be considered to evaluate the patient's requirements.

\footnotetext{
Abbreviations

ADLT: Alejandra de-la-Torre; DeCS: Descriptores en Ciencias de la Salud; EZB: Estefanía Zapata-Bravo; Emtree: EMBASE subject headings;

GRAID: German Registry of Autoimmune Diseases; GD: Graves' disease; GO: Graves ophthalmopathy; HSV: Herpes simplex virus; INF: Interferon; JMO: Juliana Muñoz-Ortiz; JRG: Juliana Reyes-Guanes; JARH: Juan Antonio Reyes-Hurtado; JBIQT: Joanna Briggs Institute Quality Tool; JVC: John Cunningham virus; LMM: Laura Mora-Muñoz; LOTG: Luis Octavio Tierradentro-García; LILACS: Literatura latinoamericana y del Caribe en Ciencias de la Salud; MS: Multiple sclerosis; MeSH: Medical Subject Headings; MGS: Marcela Gómez-Suarez; MRI: Magnetic Resonance Imaging; PRISMA: Preferred Reporting Items for Systematic Reviews and Meta-Analysis; PML: Progressive multifocal leukoencephalopathy; RRMS: Relapsing-remitting multiple sclerosis; RCT: Randomized controlled trials; SC: Subcutaneous; TEAEs: Treatment-emergent adverse events; USA: United States of America; UK: United Kingdom; VEP: Visual evoked potentials; VZV: Varicella-zoster virus; WRC: William Rojas-Carabali
}

\section{Supplementary Information}

The online version contains supplementary material available at https://doi. org/10.1186/s13643-021-01782-7.

Additional file 1. : Annex 1

Additional file 2. : Annex 2

\section{Acknowledgement}

We thank Universidad del Rosario for financing the proofreading of this manuscript and Enago (www.enago.com) for the English language review.

\section{Authors' contributions}

JMO, JRG, JARH, LMM: Management, research idea, protocol registration, protocol writing, title and abstract reading, manuscript writing and discussion, and manuscript final approval. EZB: Protocol registration, protocol writing, title and abstract reading, quality evaluation, data extraction, manuscript writing and discussion, and manuscript final approval. LOTG, WRC: Title and abstract reading, quality evaluation, data extraction, manuscript writing and discussion, and manuscript final approval. MGS: Methodology adviser. Protocol reviewing, results interpretation, and manuscript final approval. ADLT: Management, research idea, protocol writing, manuscript final approval, and thematic authority.

Funding

No funding was required to conduct this study.

\section{Availability of data and materials}

The datasets used and/or analyzed during the current study are available by the corresponding author on reasonable request.

\section{Declarations}

\section{Ethics approval}

The present study was performed according to the ethical principles for research involving human beings established by the Declaration of Helsinki, the Belmont Report, and Colombian Resolution 008430 of 1993. As it is a systematic review of the literature, it does not require approval by the ethics committee.

\section{Consent for publication}

Not applicable.

\section{Competing interests}

The authors declare that they have no competing interests.

\section{Author details}

${ }^{1}$ Neuroscience Research Group "NeURos", Escuela de Medicina y Ciencias de la Salud, Universidad del Rosario, Carrera 24 \# 63C - 69, Bogotá, Colombia. ${ }^{2}$ Escuela Barraquer Research Group, Escuela Superior de Oftalmología del Instituto Barraquer de América, Avenida Calle 100 \# 18A - 51, Bogotá, Colombia.

Received: 8 October 2020 Accepted: 28 July 2021

Published online: 28 October 2021

References

1. Kantarci $\mathrm{OH}$. Phases and phenotypes of multiple sclerosis. Continuum (Minneap Minn). 2019;25(3):636-54. https://doi.org/10.1212/CON. 0000000000000737

2. Heath G, Airody A, Gale RP. The ocular manifestations of drugs used to treat multiple sclerosis. Drugs. 2017;77(3):303-11. https://doi.org/10.1007/s40265017-0692-6.

3. Chen L, Gordon LK. Ocular manifestations of multiple sclerosis. Curr Opin Ophthalmol. 2005;16(5):315-20. https://doi.org/10.1097/01.icu.0000179804.4 9842.e2.

4. Safety Monitoring of Medicinal Products. Guidelines for setting up and running a Pharmacovigilance Centre. Uppsala Monitoring Centre - WHO Collaborating Centre for International Drug Monitoring, EQUUS, London, 2000. [Internet]. https://www.who.int/healthpromotion/about/HPG/en/. Accessed4 May 2020. 
5. Edwards IR, Aronson JK. Adverse drug reactions: definitions, diagnosis, and management. Lancet. 2000;356(9237):1255-9. https://doi.org/10.1016/S01406736(00)02799-9.

6. Moher D. Preferred reporting items for systematic reviews and metaanalyses: the PRISMA statement. Ann Intern Med. 2009;151(4):264-9, W64. https://doi.org/10.7326/0003-4819-151-4-200908180-00135.

7. Joanna Briggs Institute Global. EPB Resources \& Publications | Joanna Briggs Institute. 2021. [online] Available at: https://jbi.global/ebp. Accessed 20 May 2021.

8. Tremlett HL, Oger J. Ten years of adverse drug reaction reports for the multiple sclerosis immunomodulatory therapies: a Canadian perspective. Mult Scler. 2008;14(1):94-105. https://doi.org/10.1177/1352458507079658.

9. Calabresi PA, Radue EW, Goodin D, et al. Safety and efficacy of fingolimod in patients with relapsing-remitting multiple sclerosis (FREEDOMS II): a double-blind, randomised, placebo-controlled, phase 3 trial. Lancet Neurol. 2014;13(6):545-56. https://doi.org/10.1016/S1474-4422(14)70049-3. Erratum in: Lancet Neurol. 2013 Jun;13(6):536.

10. Cohen J, Barkhof F, Comi G, Hartung H, Khatri B, Montalban X et al. Oral Fingolimod or Intramuscular Interferon for Relapsing Multiple Sclerosis. New England Journal of Medicine. 2010;362(5):402-415. [Internet]. https://www. nejm.org/doi/full/10.1056/NEJMoa0907839. Accessed 5 May 2020.

11. Fox E, Wynn D, Cohan S, Rill D, McGuire D, Markowitz C. A randomized clinical trial of autologous T-cell therapy in multiple sclerosis: subset analysis and implications for trial design. Mult Scler. 2012;18:843-52.

12. Kappos L, Bar-Or A, Cree BAC, Fox RJ, Giovannoni G, Gold R, et al. Siponimod versus placebo in secondary progressive multiple sclerosis (EXPAND): a double-blind, randomised, phase 3 study. Lancet. 2018; 391(10127):1263-73. https://doi.org/10.1016/S0140-6736(18)30475-6.

13. Saida T, Kira Jl, Kishida S, Yamamura T, Sudo Y, Ogiwara K, et al. Efficacy, safety, and pharmacokinetics of natalizumab in Japanese multiple sclerosis patients: A double-blind, randomized controlled trial and open-label pharmacokinetic study. Mult Scler Relat Disord. 2017;11:25-31. https://doi. org/10.1016/j.msard.2016.11.002.

14. Selmaj K, Li DKB, Hartung HP, Hemmer B, Kappos L, Freedman MS, et al. Siponimod for patients with relapsing-remitting multiple sclerosis (BOLD): an adaptive, dose-ranging, randomised, phase 2 study. Lancet Neurol. 2013; 12(8):756-67. https://doi.org/10.1016/S1474-4422(13)70102-9.

15. Vermersch P, Czlonkowska A, Grimaldi LME, Confavreux C, Comi G, Kappos $L$, et al. Teriflunomide versus subcutaneous interferon beta-1a in patients with relapsing multiple sclerosis: a randomised, controlled phase 3 trial. Mult Scler. 2014;20(6):705-16. https://doi.org/10.1177/1352458513507821.

16. Voskuhl RR, Wang H, Wu TCJ, Sicotte NL, Nakamura K, Kurth F, et al. Estriol combined with glatiramer acetate for women with relapsing-remitting multiple sclerosis: a randomised, placebo-controlled, phase 2 trial. Lancet Neurol. 2016;15(1):35-46. https://doi.org/10.1016/S1474-4422(15)00322-1.

17. Viglietta V, Bourcier K, Buckle GJ, Healy B, Weiner HL, Hafler DA, et al. CTLA4lg treatment in patients with multiple sclerosis: an open-label, phase 1 clinical trial. Neurology. 2008;71(12):917-24. https://doi.org/10.1212/01.wnl. 0000325915.00112 .61

18. Pakdaman H, Abbasi M, Gharagozli K, Ashrafi F, Delavar Kasmaei H, Amini HA. A randomized double-blind trial of comparative efficacy and safety of Avonex and CinnoVex for treatment of relapsing-remitting multiple sclerosis. Neurol Sci. 2018;39(12):2107-13. https://doi.org/10.1007/s10072-01 8-3550-8.

19. Cohen JA, Comi G, Selmaj KW, Bar-Or A, Arnold DL, Steinman L, et al. Safety and efficacy of ozanimod versus interferon beta-1a in relapsing multiple sclerosis (RADIANCE): a multicentre, randomised, 24-month, phase 3 trial. Lancet Neurol. 2019;18(11):1021-33. https://doi.org/10.1016/S1474-4422(19)3 0238-8.

20. Afshar AR, Fernandes JK, Patel RD, Ksiazek SM, Sheth VS, Reder AT, et al. Cystoid macular edema associated with fingolimod use for multiple sclerosis. JAMA Ophthalmol. 2013;131(1):103-7. https://doi.org/10.1001/jama ophthalmol.2013.570.

21. Akiyama H, Suzuki $Y$, Hara D, Shinohara K, Ogura H, Akamatsu M, Hasegawa Y. Improvement of macular edema without discontinuation of fingolimod in a patient with multiple sclerosis: A case report. Med (Baltim). 2016;95(29): e4180. https://doi.org/10.1097/MD.0000000000004180.

22. Asensio-Sánchez VM, Trujillo-Guzmán L, Ramoa-Osorio R. Cystoid macular oedema after fingolimod treatment in multiple sclerosis. Arch Soc Esp Oftalmol. 2014;89(3):104-6. https://doi.org/10.1016/j.oftal.2012.07.020.
23. Bakri SJ, Swanson JW. Asymptomatic peripheral retinal hemorrhages as a manifestation of interferon beta 1a retinopathy. Semin Ophthalmol. 2015; 30(1):56-7. https://doi.org/10.3109/08820538.2013.814693.

24. Bhatti MT, Freedman SM, Mahmoud TH. Fingolimod therapy and macular hemorrhage. J Neuroophthalmol. 2013;33(4):370-2. https://doi.org/10.1097/ WNO.0b013e31829b42e1.

25. Boster AL, Nicholas JA, Topalli I, Kisanuki YY, Pei W, Morgan-Followell B, et al. Lessons learned from fatal progressive multifocal leukoencephalopathy in a patient with multiple sclerosis treated with natalizumab. JAMA Neurol. 2013;70(3):398-402. https://doi.org/10.1001/jamaneurol.2013.1960.

26. Christopher KL, Elner VM, Demirci H. Conjunctival lymphoma in a patient on fingolimod for relapsing-remitting multiple sclerosis. Ophthal Plast Reconstr Surg. 2017;33(3):e73-5. https://doi.org/10.1097/IOP.0000000000000774.

27. Chui J, Herkes GK, Chang A. Management of fingolimod-associated macular edema. JAMA Ophthalmol. 2013;131(5):694-6. https://doi.org/10.1001/jama ophthalmol.2013.47.

28. de Massougnes S, Borruat FX, Ambresin A. Peripheral bilateral telangiectasiae in multiple sclerosis patients treated with interferon b1a. Klin Monbl Augenheilkd. 2016;233(4):438-40. https://doi.org/10.1055/s-0041-111 803.

29. De Santi L, Costantini MC, Annunziata P. Long time interval between multiple sclerosis onset and occurrence of primary Sjögren's syndrome in a woman treated with interferon-beta. Acta Neurol Scand. 2005;112(3):194-6. https://doi.org/10.1111/j.1600-0404.2005.00455.x.

30. Esquenazi S. Bilateral reversible corneal edema associated with amantadine use. J Ocul Pharmacol Ther. 2009;25(6):567-70. https://doi.org/10.1089/jop.2 009.0029 .

31. Gaetani L, Menduno PS, Cometa F, Di Gregorio M, Sarchielli P, Cagini C, et al. Retinopathy during interferon- $\beta$ treatment for multiple sclerosis: case report and review of the literature. J Neurol. 2016;263(3):422-7. https://doi. org/10.1007/s00415-015-7879-0.

32. Gallego-Pinazo R, España-Gregori E, Casanova B, Pardo-López D, Díaz-Llopis $M$. Branch retinal vein occlusion during fingolimod treatment in a patient with multiple sclerosis. J Neuroophthalmol. 2011;31(3):292-3. https://doi. org/10.1097/WNO.0b013e31822bed20.

33. Fan Gaskin JC-C, Coote M. Postoperative cystoid macular oedema in a patient on fingolimod. BMJ Case Rep. 2015;2015(may12 1):bcr2015210415. https://doi.org/10.1136/bcr-2015-210415.

34. Jasani KM, Sharaf N, Rog D, Aslam T. Fingolimod-associated macular oedema. BMJ Case Rep. 2017;2017:bcr-2016. https://doi.org/10.1136/bcr-201 6-218912

35. Jeng BH, Galor A, Lee MS, Meisler DM, Hollyfield JG, Schoenfield L, et al. Amantadine-associated corneal edema potentially irreversible even after cessation of the medication. Ophthalmology. 2008;115(9):1540-4. https:// doi.org/10.1016/j.ophtha.2008.03.011.

36. Jenisch T, Dietrich-Ntoukas T, Renner AB, Helbig H, Gamulescu MA. Combined retinal artery and vein occlusions associated with interferon beta therapy. Ophthalmologe. 2012;109(1):71-5. https://doi.org/10.1007/s00347011-2448-0.

37. Kim MJ, Bhatti MT, Costello F. Famous. Surv Ophthalmol. 2016;61(4):512-9. https://doi.org/10.1016/j.survophthal.2015.12.008.

38. Lambrou GN, Kopferschmitt J, Jaeger A, Brini A. Slowly reversible central scotoma: iatrogenic effect of hyperbaric oxygenation in the treatment of multiple sclerosis. J Fr Ophtalmol. 1987;10(1):51-9.

39. Longmuir R, Lee AG, Rouleau J. Cotton wool spots associated with interferon beta-1 alpha therapy. Semin Ophthalmol. 2007;22(1):49-53. https://doi.org/10.1080/08820530601182725.

40. Mallada-Frechín J, Abellán-Miralles I, Alfaro-Beltra ML, Medrano V, Muñoz-Gil $M B$, Fernández-Izquierdo $S$, et al. Retinopathy secondary to treatment with interferon beta-1a in a patient with multiple sclerosis. Rev Neurol. 2005; 40(8):482-4. https://doi.org/10.33588/rn.4008.2005132.

41. Minuk A, Belliveau MJ, Almeida DRP, Dorrepaal SJ, Gale JS. Fingolimodassociated macular edema: resolution by sub-tenon injection of triamcinolone with continued fingolimod use. JAMA Ophthalmol. 2013; 131(6):802-4. https://doi.org/10.1001/jamaophthalmol.2013.2465.

42. Ohira M, Ito D, Shimizu T, Shibata M, Ohde H, Suzuki N. Retinopathy: an overlooked adverse effect of interferon-beta treatment of multiple sclerosis. Keio J Med. 2009;58(1):54-6. https://doi.org/10.2302/kjm.58.54.

43. Post JW, Colleaux K. Interferon beta retinopathy in a patient with multiple sclerosis. Can J Ophthalmol. 2009:44(5):e37. https://doi.org/10.3129/i09-094. 
44. Pul R, Osmanovic A, Schmalstieg H, Pielen A, Pars K, Schwenkenbecher P et al. Fingolimod associated bilateral cystoid macular edema-wait and see? [Internet]. Int J Mol Sci. 2016;17(12) https://doi.org/10.3390/ijms171221 06

45. Saatci AO, Ayhan Z, Arikan G, Sayiner A, Ada E. Unilateral acute retinal necrosis in a multiple sclerosis patient treated with high-dose systemic steroids. Int Ophthalmol. 2010;30(5):629-32. https://doi.org/10.1007/s10792010-9380-1.

46. Saito H, Suzuki M, Asakawa T, Kato S. Retinopathy in a multiple sclerosis patient undergoing interferon-therapy. Mult Scler. 2007;13(7):939-40. https://doi.org/10.1177/1352458507077403.

47. Sallansonnet-Froment M, Roux X, de Greslan T, Bounolleau P, Taillia H, Ricard D, et al. Interferon-beta retinopathy. Rev Neurol (Paris). 2009;165(11): 971-4. https://doi.org/10.1016/j.neurol.2008.11.019.

48. Schröder K, Finis D, Harmel J, Ringelstein M, Hartung HP, Geerling G, et al. Acetazolamide therapy in a case of fingolimod-associated macular edema: early benefits and long-term limitations. Mult Scler Relat Disord. 2015;4(5): 406-8. https://doi.org/10.1016/j.msard.2015.06.015.

49. Sheikh Z, Jain S, Hillen M. Acute retinal necrosis in multiple sclerosis: A neuroimmunologic challenge! Neurology. 2016;86(10):972-3. https://doi. org/10.1212/WNL.0000000000002450

50. Sommer S, Sablon JC, Zaoui M, Rozot P, Hosni A. Interferon beta-1b retinopathy during a treatment for multiple sclerosis. J Fr Ophtalmol. 2001; 24(5):509-12.

51. Spierer O, Leibovitch I. Recurrent orbital inflammation in a patient with multiple sclerosis treated with interferon-beta. Clin Exp Ophthalmol. 2011; 39(8):835-7. https://doi.org/10.1111/j.1442-9071.2011.02549.x.

52. Thoo S, Cugati S, Lee A, Chen C. Successful treatment of fingolimodassociated macular edema with intravitreal triamcinolone with continued fingolimod use. Mult Scler. 2015;21(2):249-51. https://doi.org/10.1177/13524 58514528759.

53. Trinh T, Haridas AS, Sullivan TJ. Ocular findings in alemtuzumab (Campath1H)-induced thyroid eye disease. Ophthal Plast Reconstr Surg. 2016;32(6): e128-9. https://doi.org/10.1097//OP.0000000000000310.

54. Turaka K, Bryan JS. Does fingolimod in multiple sclerosis patients cause macular edema? J Neurol. 2012;259(2):386-8. https://doi.org/10.1007/s0041 5-011-6367-4.

55. Ueda N, Saida K. Retinal hemorrhages following fingolimod treatment for multiple sclerosis; a case report. BMC Ophthalmol. 2015;15(1):135. https:// doi.org/10.1186/s12886-015-0125-9.

56. Zecca C, Nessi F, Bernasconi E, Gobbi C. Ocular toxoplasmosis during natalizumab treatment. Neurology. 2009;73(17):1418-9. https://doi.org/1 0.1212 WNL.0b013e3181bd114f.

57. Cifuentes-Canorea P, Nieves-Moreno M, Sáenz-Francés F, Santos-Bueso E. Edema macular precoz y recurrente en paciente en tratamiento con fingolimod. Neurología. 2019;34(3):206-7. https://doi.org/10.1016/j.nrl.2016. 08.005.

58. Husmann R, Davies JB, Ghannam M, Berry B, Kelkar P. Fingolimod-associated macular edema controlled with nepafenac non-steroidal anti-inflammatory opthalmologic applications. Clin Mol Allergy. 2020;18(1):3. https://doi.org/1 0.1186/s12948-020-00119-4.

59. Tsourdi E, Gruber M, Rauner M, Blankenburg J, Ziemssen T, Hofbauer LC. Graves' disease after treatment with alemtuzumab for multiple sclerosis. Hormones (Athens). 2015;14(1):148-53. https://doi.org/10.14310/horm.2002.1 501.

60. Williams KE, Johnson LN. Neuroretinitis in patients with multiple sclerosis. Ophthalmology. 2004;111(2):335-40; discussion 340-1. https://doi.org/10.101 6/S0161-6420(03)00663-8.

61. Roos JCP, Moran C, Chatterjee VK, Jones J, Coles A, Murthy R. Immune reconstitution after alemtuzumab therapy for multiple sclerosis triggering Graves' orbitopathy: a case series. Eye (Lond). 2019;33(2):223-9. https://doi. org/10.1038/s41433-018-0282-1.

62. Holmén C, Piehl F, Hillert J, Fogdell-Hahn A, Lundkvist M, Karlberg E, et al. A Swedish national post-marketing surveillance study of natalizumab treatment in multiple sclerosis. Mult Scler. 2011;17(6):708-19. https://doi. org/10.1177/1352458510394701.

63. Lapierre $Y, O^{\prime}$ Connor P, Devonshire V, Freedman MS, Kremenchutzky M, Yeung $M$, et al. Canadian experience with fingolimod: adherence to treatment and monitoring. Can J Neurol Sci. 2016;43(2):278-83. https://doi org/10.1017/cjn.2015.325
64. Laroni A, Brogi D, Brescia Morra V, Guidi L, Pozzilli C, Comi G, et al. Safety and tolerability of fingolimod in patients with relapsing-remitting multiple sclerosis: results of an open-label clinical trial in Italy. Neurol Sci. 2017;38(1): 53-9. https://doi.org/10.1007/s10072-016-2701-z.

65. Liscić RM, Brecelj J. Visual evoked potentials in multiple sclerosis patients treated with interferon beta-1a. Croat Med J. 2004:45(3):323-7.

66. Makioka H, Nakaya F, Ling Y, Torii S, Saida T, Kira Jl. Safety and effectiveness of interferon $\beta$-1a intramuscular therapy: results of the postmarketing drug surveillance in Japan. Rinsho Shinkeigaku. 2017;57(10):553-61. https://doi. org/10.5692/clinicalneurol.cn-001074.

67. Ontaneda D, Hara-Cleaver C, Rudick RA, Cohen JA, Bermel RA. Early tolerability and safety of fingolimod in clinical practice. J Neurol Sci. 2012; 323(1-2):167-72. https://doi.org/10.1016/j.jns.2012.09.009.

68. Rommer PS, Dörner T, Freivogel K, Haas J, Kieseier BC, Kümpfel T, et al. Safety and clinical outcomes of rituximab treatment in patients with multiple sclerosis and neuromyelitis optica: experience from a national online registry (GRAID). J Neuroimmune Pharmacol. 2016;11(1):1-8 https:// doi.org/10.1007/s11481-015-9646-5, 1

69. Smith MY, Sabidó-Espin M, Trochanov A, Samuelson M, Guedes S, Corvino FA, et al. Postmarketing safety profile of subcutaneous interferon beta-1a given 3 times weekly: A retrospective administrative claims analysis. J Manag Care Spec Pharm. 2015;21(8):650-60. https://doi.org/10.18553/jmcp.2 015.21.8.650.

70. Tuohy O, Costelloe L, Hill-Cawthorne G, Bjornson I, Harding K, Robertson N, et al. Alemtuzumab treatment of multiple sclerosis: long-term safety and efficacy. J Neurol Neurosurg Psychiatry. 2015;86(2):208-15. https://doi.org/1 0.1136/jnnp-2014-307721.

71. Weglinski L, Manceau P, Thomas-Pohl M, Le Breton F, Amarenco G. Prospective evaluation of mouth and eye dryness induced by antimuscarinic drugs used for neurogenic overactive bladder in 35 patients with multiple sclerosis. Prog Urol. 2017;27(4):253-60. https://doi.org/10.1016/ j.purol.2016.10.005.

72. Willis MD, Harding KE, Pickersgill TP, Wardle M, Pearson OR, Scolding NJ, et al. Alemtuzumab for multiple sclerosis: long term follow-up in a multicentre cohort. Mult Scler. 2016;22(9):1215-23. https://doi.org/10.1177/13524 58515614092

73. Zarbin MA, Jampol LM, Jager RD, Reder AT, Francis G, Collins W, et al. Ophthalmic evaluations in clinical studies of fingolimod (FTY720) in multiple sclerosis. Ophthalmology. 2013;120(7):1432-9. https://doi.org/10.1016/j. ophtha.2012.12.040

74. Gugliandolo A, Longo F, Marrosu MG, Mancardi GL, Gandoglia I, Melis M, et al. A multicentric pharmacovigilance study: collection and analysis of adverse drug reactions in relapsing-remitting multiple sclerosis patients [Internet]. Ther Clin Risk Manag. 2018;14:1765-88. https://doi.org/10.2147/ TCRM.S174864.

75. Fruschelli M, Capozzoli M, Gelmi MC, Masi G, Annunziata P. Longitudinal quantitative assessment of macula during therapy with fingolimod in relapsing-remitting multiple sclerosis. Int Ophthalmol. 2019;39(4):777-81. https://doi.org/10.1007/s10792-018-0870-x.

76. Lim LL, Silva DG, Lo TC, Pimentel RS, Butzkueven H, Hall AJ. Uveitis in patients with multiple sclerosis in clinical trials of fingolimod: incidence, prevalence, and impact on disease course. Ophthalmology. 2019;126(3):43844. https://doi.org/10.1016/j.ophtha.2018.10.013.

77. Comi G, Kappos L, Selmaj KW, Bar-Or A, Arnold DL, Steinman L, et al. Safety and efficacy of ozanimod versus interferon beta-1a in relapsing multiple sclerosis (sunbeam): a multicentre, randomised, minimum 12-month, phase 3 trial. Lancet Neurol. 2019;18(11):1009-20. https://doi.org/10.1016/S14 74-4422(19)30239-X

78. Brown BA. Natalizumab in the treatment of multiple sclerosis. Ther Clin Risk Manag. 2009:5(3):585-94. https://doi.org/10.2147/tcrm.55218.

79. Polman $\mathrm{CH}, \mathrm{O}^{\prime}$ Connor PW, Havrdova E, Hutchinson M, Kappos L, Miller DH, et al. A randomized, placebo-controlled trial of natalizumab for relapsing multiple sclerosis. N Engl J Med. 2006;354(9):899-910. https://doi.org/10.1 056/NEJMoa044397.

80. Zorzela L, Loke YK, loannidis JP, Golder S, Santaguida P, Altman DG, et al. PRISMA harms checklist: improving harms reporting in systematic reviews. BMJ. 2016;352:1157. https://doi.org/10.1136/bmj.i157.

81. Peryer G, Golder S, Junqueira D, Vohra S, Loke YK. Chapter 19: Adverse effects. In: Higgins JPT, Thomas J, Chandler J, Cumpston M, Li T, Page MJ, Welch VA (editors). Cochrane Handbook for Systematic Reviews of 
Interventions version 6.2 (updated February 2021). Cochrane, 2021. Available from https://www.training.cochrane.org/handbook.

82. Management Sciences for Health and World Health Organization. Drug and Therapeutics Committee Training Course. Submitted to the U.S. Agency for International Development by the Rational Pharmaceutical Management Plus Program. Arlington: Management Sciences for Health; 2007.

83. Miller DH, Chard DT, Ciccarelli O. Clinically isolated syndromes. Lancet Neurol. 2012;11(2):157-69. https://doi.org/10.1016/S1474-4422(11)70274-5.

84. Frohman TC, Graves J, Balcer L, Galetta SL, Frohman EM. The neuroophthalmology of multiple sclerosis. Continuum (Minneap Minn). 2010;16(5 Multiple Sclerosis):122-46 https://doi.org/10.1212/01.CON.0000389938.07532. 67.

85. Bomprezzi R, Powers JM. IFN- $\beta 1$ b may severely exacerbate Japanese opticspinal MS in neuromyelitis optica spectrum. 2020. Accessed 5 May 2020. https://n.neurology.org/content/ifn\%CE\%B2-1b-may-severely-exacerbate-ja panese-optic-spinal-ms-neuromyelitis-optica-spectrum.

86. Pula JH, Reder AT. Multiple sclerosis. Part I: Neuro-ophthalmic manifestations. Curr Opin Ophthalmol. 2009;20(6):467-75. https://doi.org/1 0.1097/ICU.0b013e328331913b

87. Andrew L. Ophthalmic screening recommendation for new MS drug. [online] The Ophthalmic News and Education Network. 2018. Available at: https://www.aao.org/editors-choice/ophthalmic-screening-recommendationnew-ms-drug. Accessed 20 May 2021

88. Wiersinga WM, Smit T, van der Gaag R, Koornneef L. Temporal relationship between onset of Graves' ophthalmopathy and onset of thyroidal Graves' disease. J Endocrinol Invest. 1988:11(8):615-9. https://doi.org/10.1007/BF033 50193.

89. Buonomo AR, Zappulo E, Viceconte G, Scotto R, Borgia G, Gentile I. Risk of opportunistic infections in patients treated with alemtuzumab for multiple sclerosis. Expert Opin Drug Saf. 2018;17(7):709-17.[Editor1]. https://doi.org/1 $0.1080 / 14740338.2018 .1483330$

\section{Publisher's Note}

Springer Nature remains neutral with regard to jurisdictional claims in published maps and institutional affiliations.

Ready to submit your research? Choose BMC and benefit from:

- fast, convenient online submission

- thorough peer review by experienced researchers in your field

- rapid publication on acceptance

- support for research data, including large and complex data types

- gold Open Access which fosters wider collaboration and increased citations

- maximum visibility for your research: over $100 \mathrm{M}$ website views per year

At $\mathrm{BMC}$, research is always in progress.

Learn more biomedcentral.com/submissions 